\title{
Inteligencia Emocional y Empatía: su influencia en la Competencia Social en Educación Secundaria Obligatoria ${ }^{1}$
}

\section{Emotional Intelligence and Empathy: their influence on the Social Competence in Secondary Obligatory Education}

\author{
Raquel Gilar Corbí2, Pablo Miñano Pérez, Juan Luis Castejón Costa \\ Universidad de Alicante, España
}

(Recepción: Marzo 2008 - Aceptación: Mayo 2008)

\begin{abstract}
Resumen
La finalidad del presente trabajo es analizar las relaciones existentes entre la competencia social y la inteligencia emocional en estudiantes de Educación Secundaria Obligatoria. Así mismo, también nos proponemos analizar el papel de la empatía y sus relaciones con la competencia social y la inteligencia emocional, ya que en la mayoría de los estudios existentes aparece relacionada de un modo u otro con estos constructos. Los participantes en nuestro estudio fueron 110 estudiantes de primer ciclo de ESO. Los instrumentos empleados fueron: el test EQ-i:YV de BarOn (1997), el Cuestionario Matson de habilidades sociales para adolescentes (Matson, Rotatori, y Helsel, 1983) y el Interpersonal Reactivity Index, IRI (Davis, 1983). Las técnicas de análisis de datos incluyen: análisis de correlación, análisis de regresión múltiple paso a paso y análisis de regresión múltiple de tipo jerárquico. Los resultados obtenidos nos permiten identificar un conjunto de variables que están directamente implicadas en la Competencia social. Palabras clave: Inteligencia emocional, Competencia social, Empatía.
\end{abstract}

\begin{abstract}
The purpose of the present paper is to analyse the current connections between the social competence and the emotional intelligence in students of Secondary Obligatory Education. Likewise, we also propose to analyse the empathy's role and its connections with the social competence and the emotional intelligence, given that it appears related in most of the existing studies, in one way or another, to these constructs. The participants in our study were 110 students of the first period of Secondary Obligatory Education. The utilized means were: the test EQ-i:YV, by Baron (1997), the Questionnaire Matson of social abilities for adolescents (Matson, Rotatori, and Helsel, 1983) and Interpersonal Reactivity Index, IRI (Davis, 1983). The analysis of information's techniques include: correlation analysis, analyse of multiple regression step by step and analyse of multiple regression of hierarchical type. The obtained results allow us to identify a group of variables that are directly involved in the social competence.
\end{abstract}

Key words: Emotional intelligence, Social competence, Empathy.

1 El presente estudio ha sido subvencionado por la Consellería de Educación y Ciencia de la Generalitat Valenciana, España, mediante el Proyecto GV07/181 concedido a la primera autora.

2 Correspondencia a: Raquel Gilar Corbí. Departamento de Psicología Evolutiva y Didáctica, Universidad de Alicante. Campus de San Vicente del Raspeig. Ap. Correos 99, 03080 Alicante. E-mail: raquel.gilar@ua.es

Teléfono: 965903861 


\section{Introducción}

La finalidad del presente trabajo es analizar las relaciones existentes entre la competencia social y la inteligencia emocional en estudiantes de Educación Secundaria Obligatoria.

Así mismo, también nos proponemos analizar el papel de la empatía y sus relaciones con la competencia social y la inteligencia emocional, ya que en la mayoría de los estudios existentes aparece relacionada de un modo u otro con estos constructos. Boyatzis (2005) plantea que, situadas en el plano del reconocimiento de las emociones, la auto-conciencia (como parte de la competencia personal), junto con la empatía (como parte de la competencia social), se reflejan en las habilidades sociales, estando éstas situadas en el plano de la regulación. Nos proponemos por tanto, establecer de modo preciso, el tipo de relación y en qué sentido se produce esa relación entre estos tres conceptos.

Existen numerosos estudios que señalan la utilidad potencial de la inteligencia emocional en el contexto académico y de la necesidad de integrar la enseñanza emocional en las escuelas (Elias, Chan, Caputi, 2000; Gil-Olarte, Palomera y Brackett, 2006; Lopes y Salovey, 2004; Pool, 1997). Estos trabajos analizan el papel de la emoción y la regulación emocional en los problemas de comportamiento y la competencia social (Eisenberg y Fabes, 2006; Loukas y Prelow, 2004; Ortega, 1997; Ortega y Lera, 2000), y señalan su importancia como factor preventivo de comportamientos conflictivos y de mejora de los procesos emocionales implícitos en los contextos interpersonales (Guil, Gil-Olarte, Mestre y Núñez, 2006; Mestre, Palmero y Guil, 2004).

Debido a la naturaleza de nuestro estudio necesitamos partir de una delimitación conceptual de los distintos términos implicados.

En cuanto a la inteligencia emocional, existen dos concepciones diferentes. Por un lado, la propuesta por autores como Bar-On (2000), Goleman $(1995,1998)$ y McCrae (2000), que consideran la inteligencia emocional como combinación de una serie de atributos relacionados de forma muy estrecha con la personalidad, distinta del CI, y relacionada con competencias referentes al logro académico. Mientras que Mayer, Caruso y Salovey (2000), Mayer, Caruso, Salovey y Sitarenios (2003), plantean un concepto de inteligencia emocional que la define como capacidad para percibir y entender información emocional.

Salovey y Mayer (1990) plantean el modelo de inteligencia emocional que comprende 5 capacidades: el conocimiento de las propias emociones, el control emocional, la capacidad de motivarse a uno mismo, la empatía y las habilidades sociales. Las tres primeras capacidades harían referencia al área de la inteligencia intrapersonal y las dos últimas, al área de la inteligencia interpersonal.

Consideramos importante señalar que la evidencia científica del concepto de inteligencia emocional, tal como plantean diversas revisiones, aún es escasa (Mathews, Zeidner y Roberts, 2003; Zeidner, Mathews y Roberts, 2004), existiendo problemas conceptuales y empíricos aún sin resolver (Schulze y Roberts, 2005).

El término competencia define un conjunto de habilidades comprometidas en el rendimiento y en la resolución de problemas del ámbito personal o profesional (García, 2003; Le Boterf, 2001; LevyLeboyer, 1997), mientras que la inteligencia se refiere a los subcomponentes de estas habilidades presentes en las formas generales del pensamiento y el entendimiento (Sternberg, Castejón, Prieto, Hautamäki y Grigorenko, 2000). Topping, Bremner y Holmes (2000), definen el concepto de competencia social como la posesión y uso de la habilidad para integrar pensamiento, sentimiento y comportamiento, para conseguir éxito en los comportamientos y tareas sociales valorados en el contexto cultural en que se encuentren. La competencia social no es un atributo homogéneo, sino una constelación de pensamientos, sentimientos, habilidades y comportamientos que varían de una situación a otra. Asimismo, señalan que en el escenario escolar, esos comportamientos y tareas deberían incluir el acceso con éxito al currículum escolar, asociar las necesidades personales y sociales, y el desarrollo de habilidades y actitudes transferibles más allá de la escuela. 
Zigler y Trickett (1978) destacan dos aspectos de la competencia social: primero, percibir qué tipo de comportamiento es necesario en una determinada situación, y segundo, es necesario que la persona posea determinadas características como auto-estima y auto-eficacia.

Kliewer (1991) señala que los niños socialmente competentes son hábiles para entender las normas sociales, para interaccionar con los iguales y con los adultos y para regular sus emociones, especialmente las negativas. En esta misma línea, Del Prette y Del Prette(1999) definen la competencia social como la capacidad de conseguir los objetivos de una situación interpersonal, manteniendo una relación con el interlocutor a través del equilibrio de poder y de relaciones positivas. Del mismo modo, Tyler (1984) define competencia social como el modo en que un individuo interacciona con los acontecimientos de su vida, tanto en el sentido de la resolución de problemas como de autorrealización. Dentro de esta misma perspectiva, un individuo competente es capaz de demostrar sus potencialidades y sentimientos positivos en relación a sí mismo, es decir, es capaz de establecer metas y trazar estrategias para conseguir buenos resultados incluso cuando fracasa (Cecconello, 1999).

Coble, Grantt y Malinckrodt (1996) destacan otro aspecto importante de la competencia social, la capacidad de la persona para confiar en sus semejantes y utilizar sus habilidades para establecer relaciones interpersonales. La habilidad del sujeto para ejercer nuevos papeles y adecuar su comportamiento y actitudes a otros contextos es considerada un aspecto importante de la competencia social, relacionada con la adaptación a distintos ambientes (Cecconello y Koller, 2000). Un niño socialmente competente es capaz de ser sensible y empático con sus padres, de implicarse en actividades sociales positivas, de formar relaciones de amistad y de adaptarse a situaciones de estrés (Howes, Matheson y Hamilton, 1994).

La socialización de las emociones es un aspecto de central importancia para la expresividad emocional y la empatía. Para algunos autores, los niños tienden a comportarse de una manera constructiva y demostrar competencia social cuando aprenden a regular sus emociones (Eisenberg, Fabes y Murphy, 1996).

Caruso y Mayer (1998) recogen tres perspectivas en la definición de empatía: 1. Empatía cognitiva, en relación con la toma de perspectiva o la comprensión de los otros. Hogan (1969) describe la empatía como la comprensión intelectual o imaginativa de la condición o estado de los otros sin la experiencia real de los sentimientos de la persona; 2. Empatía como activación emocional o simpatía en respuesta a los sentimientos o experiencias de otros. Mehrabian y Epstein (1972) definen la empatía como la responsabilidad aumentada hacia la experiencia emocional de otros; 3 . Empatía desde una perspectiva integrada, que emplea las perspectivas emocional y cognitiva en el estudio de la empatía. Empatía como un constructo multidimensional (Davis, 1983; Thornton y Thornton, 1995).

En la literatura existente aparecen gran cantidad de estudios que ponen de manifiesto la relación, en mayor o menor grado, entre los constructos analizados en nuestro estudio, inteligencia emocional, competencia social y empatía (Extremera y Fernández-Berrocal, 2004; Garmez, 1996; Goleman, 2005; Boyatzis, 2005; Howes et al., 1994; Juntilla, Voeten, Kaukiainen y Vauras, 2006; Miville, Carlozzi, Gushue, Schara y Veda, 2006; Muncer y Ling, 2006; Simco y Joan, 2000), haciéndose necesario establecer de forma precisa las relaciones existentes entre ellos.

Varios indicios apuntan a una relación entre empatía y competencia social (Garmez, 1996; Howes et al., 1994). Juntilla et al. (2006) plantean una medida de la competencia social en niños donde la empatía se presenta como una de las subdimensiones de la conducta prosocial en la competencia social. Simco y Joan (2000) encuentran una correlación significativa entre competencia social y empatía, en el sentido de que los niños con niveles inferiores de competencia social tienden a mostrar niveles inferiores de empatía.

Por otra parte, existen considerables coincidencias entre algunos de los conceptos claves de inteligencia emocional y empatía (Goleman, 2005; Boyatzis, 2005; Muncer y Ling, 2006). Petrides y Furnham (2001) han identificado el manejo emocional de los otros, la percepción de la emoción, las 
habilidades de relación, la competencia social y la empatía como elementos claves de la Inteligencia Emocional. Extremera y Fernández-Berrocal (2004) encontraron relaciones significativas entre distintos aspectos de la Inteligencia Emocional y la empatía. Así mismo, Miville et al. (2006) señalan que el análisis de regresión jerárquica al que sometieron los datos de su estudio indicó que la inteligencia emocional, junto con el género, contribuyó significativamente a la explicación de las puntuaciones logradas en empatía.

El objetivo de nuestro estudio es, por tanto, establecer las relaciones entre la inteligencia emocional, la empatía y la competencia social, así como determinar la influencia de la inteligencia emocional y la empatía sobre la competencia social, considerando ésta como un constructo multidimensional.

\section{Método}

\section{Participantes}

Los participantes en este trabajo son 110 estudiantes españoles de primer ciclo de Educación Secundaria Obligatoria con edades comprendidas entre los 13 y 14 años (La Educación Secundaria Obligatoria en España es una etapa educativa obligatoria y gratuita que completa la educación básica, consta de cuatro cursos divididos en dos ciclos de dos cursos cada uno), el 49,4\% cursan $1^{\circ}$ y el $50,6 \%$ cursan $2^{\circ}$ curso). El 51,2\% son chicos y el 48,8\% chicas.

\section{Variables e instrumentos}

Los instrumentos empleados fueron:

a) La medida del Cociente Emocional, versión para jóvenes (EQ-i: YV) de Bar-On (1977, 2000); que nos da información sobre cuatro aspectos de la inteligencia emocional: factor interpersonal, factor intrapersonal, factor de manejo de estrés y factor de adaptabilidad; así como una puntuación total de la inteligencia emocional. Ugarriza (2001) encuentra un coeficiente de consistencia interna para el cuestionario total de $\alpha=.93$.

b) El Cuestionario Matson de Habilidades Sociales para Adolescentes (Matson, Rotatori, y Helsel, 1983, 1983), que evalúa el grado de adecuación de la conducta social. Nos da información sobre cuatro factores: Agresividad/Conducta antisocial, Habilidades sociales/Asertividad, Engreimiento/Arrogancia, Soledad/Ansiedad social, y una puntuación Total de Inadecuación Social. Esta puntuación en Inadecuación Social es la que emplearemos como criterio para la explicación de la competencia social. El coeficiente de consistencia interna para la puntuación total es de $\alpha=.88$ y para los diferentes factores, los coeficientes de consistencia interna oscilan entre $\alpha=.63$ y $\alpha=.91$ (Méndez, Hidalgo e Inglés, 2002).

c) Una medida de la empatía, el Interpersonal Reactivity Index, IRI (Davis, 1983), en su adaptación española realizada por Pérez-Abéniz, de Paúl, Etxebarría, Montes y Torres (2003). El cuestionario evalúa la disposición empática a través de cuatro factores, dos cognitivos y dos emocionales: Toma de Perspectiva; Fantasía; Preocupación Empática; Malestar Personal. BaronCohen y Wheelwright (2004), tras realizar una amplia revisión de los métodos existentes para medir la empatía, señalan como la más adecuada la formulada por Davis (1983). Los coeficientes de consistencia interna variaban de $\alpha=.70$ a $\alpha=.78$ (Pérez-Albéniz, De Paúl, Etxeberría, Montes y Torres, 2003).

\section{Procedimiento}

En un primer momento se solicitó la participación del Centro Educativo, exponiendo los objetivos de la investigación, los beneficios esperados y el tipo de colaboración que se solicita del Centro. Del mismo modo, se solicitó, tras una sesión explicativa, el consentimiento de los padres, mediante un documento firmado, para que sus hijos participen en el estudio. 
Los datos referentes a las distintas medidas empleadas se recogieron en las sesiones de tutoría. En primer lugar obtuvimos los datos referentes a la medida de empatía (IRI), en una segunda sesión se recogieron los datos referentes a la medida de la inteligencia emocional (EQ-i) y en una tercera sesión se obtuvieron los datos referentes a la competencia social (Matson).

\section{Diseño y Análisis de datos}

Los objetivos propuestos y el procedimiento seguido requiere el uso de un diseño correlacional. Como técnicas de análisis estadístico se ha empleado el coeficiente de correlación de Pearson, la regresión múltiple siguiendo el método paso a paso y la regresión jerárquica.

Con la técnica de regresión múltiple se puede establecer la contribución independiente de cada variable, a la predicción/explicación del criterio, una vez separada la parte de varianza común que tiene con otras variables predictoras.

Para establecer el valor predictivo de las variables consideradas en el trabajo sobre la competencia social, se llevan a cabo una serie de análisis de regresión múltiple, tomando como variable criterio la Inadecuación social (Matson).

Existen distintos tipos de análisis de regresión múltiple dependiendo de los métodos estadísticos de partición de la varianza del criterio empleados y de las asunciones teóricas que se realicen (Castejón y Navas, 1992; Cohen y Cohen, 1983; Darlington, 1990). Dos de los procedimientos de análisis más empleados son el análisis de regresión múltiple "paso a paso" (stepwise) de selección de variables y el método jerárquico. El método jerárquico permite establecer la contribución específica de alguna/as variables, una vez controlado el efecto de las variables introducidas anteriormente en la ecuación.

En el método de análisis de regresión jerárquico o método de partición incremental de la varianza, se necesita partir de un modelo teórico previo que nos indique el orden de entrada de las variables en la ecuación de regresión.

En este tipo de análisis, a las variables que entran primero en la ecuación se les atribuye toda la varianza que estas puedan explicar por sí mismas y por la contribución común que compartan con las variables que se incluyen después. De modo que, cada variable adicional incluye la varianza que ésta tiene en común con las variables que se introducen después. De esta forma, las variables que entran primero actúan como covariantes de las que se incluyen posteriormente, pudiendo así parcializar el efecto de las primeras sobre las segundas. Sólo la última variable introducida contribuye de forma única a la explicación del criterio. Se produce así, una sobreestimación del valor predictivo de las variables que entran primero en la ecuación, por lo que la decisión de introducir inicialmente unas variables u otras debe hacerse sobre una base teórica.

Aparte de la posibilidad de examinar la contribución única de las variables o conjuntos de variables que se introducen posteriormente en la ecuación, el procedimiento de análisis de regresión jerárquica permite establecer si se produce un incremento significativo en la varianza explicada del criterio, cada vez que se introduce una variable o bloque de variables en la ecuación.

Para el análisis de datos se ha empleado el paquete estadístico SPSS-Versión 15.

\section{Resultados}

\section{Análisis correlacionales}

En la tabla 1 se muestran las correlaciones entre las variables estudiadas.

De los análisis de correlación se desprende que existen correlaciones significativas entre los diferentes factores de cada uno de los cuestionarios empleados, como cabía esperar. 
Aparecen correlaciones significativas entre los factores Toma de perspectiva y Fantasía $(r=.340$, $\mathrm{p}=.01)$, entre Toma de perspectiva y Preocupación empática $(\mathrm{r}=.510, \mathrm{p}=.01)$ y entre Fantasía y Preocupación empática ( $\mathrm{r}=.507, \mathrm{p}=.01$ ), todos ellos factores integrantes del Interpersonal Reactivity Index.

También aparecen correlaciones significativas entre los factores de la medida de la competencia social, el cuestionario Matson, Agresividad y Arrogancia ( $\mathrm{r}=.491, \mathrm{p}=.01)$, y entre Inadecuación social y Agresividad ( $r=.791, p=.01)$, Habilidades sociales $(r=-.747, p=.01)$, y Arrogancia $(r=.474$, $\mathrm{p}=.01)$.

Asimismo, encontramos correlaciones significativas entre algunos de los factores integrantes de la medida de inteligencia emocional, EQ-i YV como entre los factores de Adaptabilidad a Interpersonal $(\mathrm{r}=.444, \mathrm{p}=.01)$ y el Total EQ-i y los cuatro factores considerados del EQ-i, Factor Intrapersonal $(\mathrm{r}=.644, \mathrm{p}=.01)$, Factor Interpersonal $(\mathrm{r}=.776, \mathrm{p}=.01)$, Manejo del estrés $(\mathrm{r}=.418$, $\mathrm{p}=.01)$ y Adaptabilidad $(\mathrm{r}=.642, \mathrm{p}=.01)$.

\section{Tabla 1. Correlaciones entre variables}

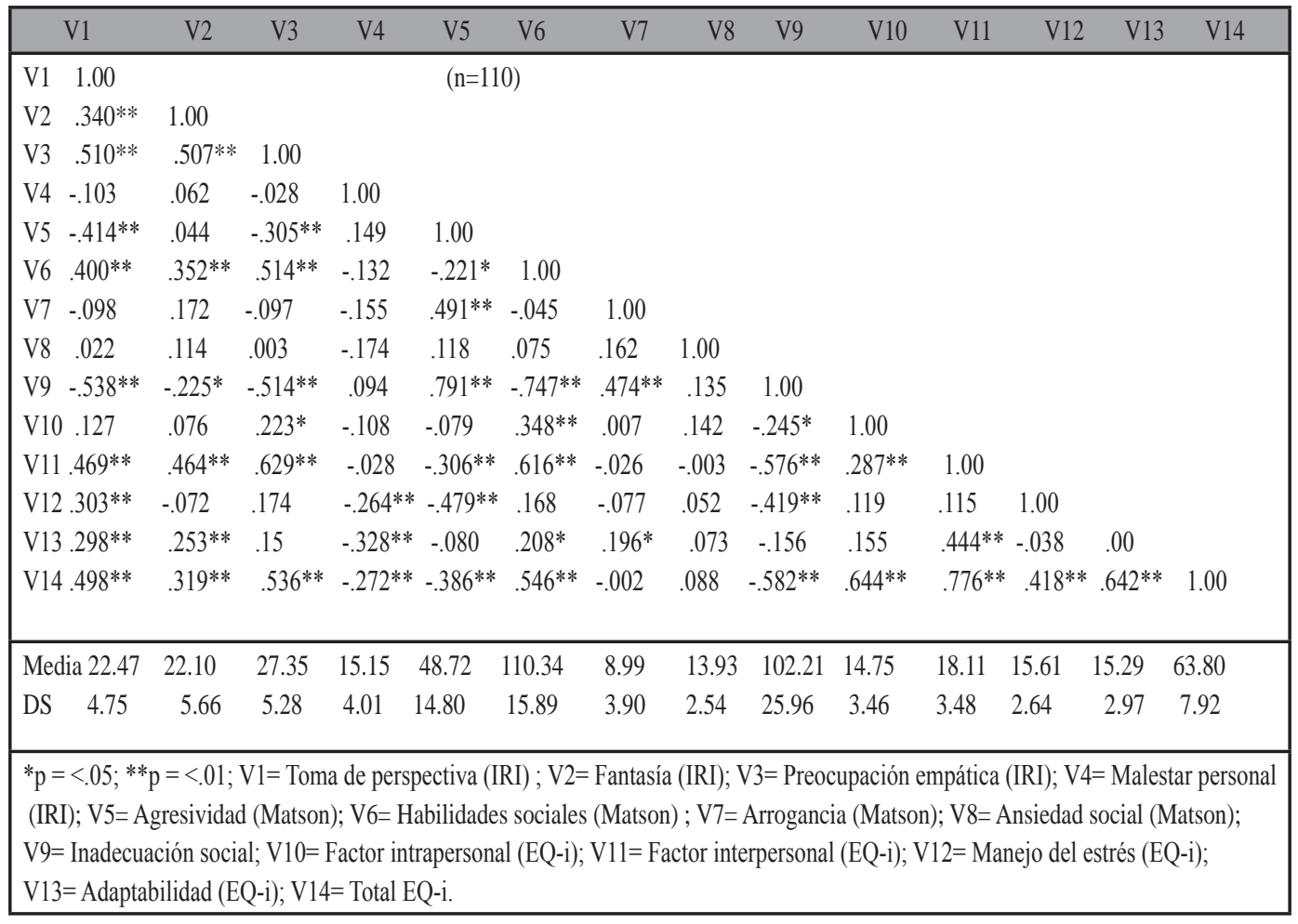

También encontramos relaciones entre aspectos de la medida de empatía y de la inteligencia emocional: el factor Preocupación empática (IRI) correlaciona con el Factor Interpersonal ( $\mathrm{r}=$.629, $\mathrm{p}=.01)$ y con el Total EQ-i $(\mathrm{r}=.536, \mathrm{p}=.01)$. Del mismo modo, hallamos correlaciones significativas entre los factores integrantes de la medida de la competencia social y las medidas de empatía e inteligencia emocional. El factor Habilidades sociales (Matson) correlaciona con el Factor Interpersonal (EQ-i) $(\mathrm{r}=.616, \mathrm{p}=.01)$, con el Toatl EQ-i $(\mathrm{r}=.546, \mathrm{p}=.01)$ y con la Preocupación empática (IRI) $(\mathrm{r}=.514, \mathrm{p}=.01)$. Y la puntuación en Inadecuación Social correlaciona de forma negativa con los factores Toma de perspectiva (IRI) $(\mathrm{r}=-.538, \mathrm{p}=.01)$, Preocupación empática (IRI) $(\mathrm{r}=-.514, \mathrm{p}=.01)$, Factor Interpersonal (EQ-i) $(\mathrm{r}=-.576, \mathrm{p}=.01)$ y Total EQ-i $(\mathrm{r}=-.582, \mathrm{p}=.01)$.

De este modo, las variables que mantienen correlaciones significativas con la medida de la 
competencia social son el Factor interpersonal (EQ-i), el Total EQ-i, la Toma de perspectiva (IRI) y la Preocupación empática (IRI).

No obstante, las correlaciones bivariadas entre el rendimiento y las variables enunciadas no son suficientes para establecer la utilidad de estas variables para predecir y/o explicar el rendimiento, ya que estas variables pueden ser redundantes entre sí.

Análisis de regresión paso a paso

El método de regresión paso a paso, tiene en cuenta la correlación entre las variables y es útil para seleccionar, a partir de un conjunto amplio, aquellas variables que hacen una contribución relativamente independiente a la predicción del criterio.

En la Tabla 2 se presentan los resultados de los análisis de regresión paso a paso tomando como criterio la inadecuación social.

Tabla 2. Resumen del análisis de regresión paso a paso para las variables predictoras de la Inadecuación Social. $(\mathrm{N}=110)$

\begin{tabular}{|c|ccc|}
\hline Variable & $\mathrm{B}$ & $\beta$ & $\mathrm{t}$ \\
\hline & & & \\
Preocupación empática & -1.21 & $-.23^{*}$ & -1.95 \\
Manejo del estrés & -2.51 & $-.25^{*}$ & -2.81 \\
Interpersonal & -2.37 & $-.29^{*}$ & -2.69 \\
Toma de perspectiva & -1.28 & $-.23^{*}$ & -2.16 \\
\hline
\end{tabular}

Nota. $\mathrm{R}=.76 ; \mathrm{R}^{2}=.32 ; \mathrm{F}=21.15(\mathrm{ps}<.000)$.

${ }^{*} \mathrm{p}<.05$

La variable que hace una mayor contribución a la explicación de la varianza del criterio es el Factor interpersonal (EQ-i) $(\beta=-.29, p=.009)$. También contribuyen a explicar de forma significativa la inadecuación social, el factor Manejo del estrés (EQ-i) $(\beta=-.25, p=.007)$, la Toma de perspectiva (IRI) $(\beta=-.23, p=.035)$ y la Preocupación empática (IRI) $(\beta=-.23, p=.05)$. Como vemos todos los valores son negativos, una vez que se ponen en relación con la Inadecuación Social, considerada como criterio.

Así pues, las variables que adquieren mayor relevancia predictiva están relacionadas tanto con la inteligencia emocional como con la empatía.

Análisis de regresión jerárquica.

En la tabla 3 aparecen los resultados referentes a los análisis de regresión múltiple de tipo jerárquico donde han sido introducidas las variables tomando como criterio la Inadecuación social.

En primer lugar se introduce en la ecuación el bloque de variables relativas al cuestionario sobre empatía. La contribución de este bloque es altamente significativa $(\mathrm{F}=16.52, \mathrm{p}=.000)$. Con la inclusión del segundo bloque de variables relativas, la medida de la inteligencia emocional vuelve a producirse un aumento significativo de la varianza explicada $(\mathrm{F}=11.40, \mathrm{p}=.000)$. 
Tabla 3. Resumen del análisis de regresión de tipo jerárquico para las variables predictoras de la Inadecuación Social $(\mathrm{N}=\mathbf{1 1 0})$

\begin{tabular}{|c|c|c|c|}
\hline Variables & B & SEB & $\beta$ \\
\hline Paso 1 & & & \\
Iri toma de perspectiva & -2.076 & .642 & $-.363^{* *}$ \\
Iri fantaśa & .740 & .447 & .164 \\
Iri preocupación empática & -2.295 & .624 & $-.437^{* *}$ \\
Paso 2 & & & \\
Iri toma de perspectiva & -1.322 & .628 & $-.321^{*}$ \\
Iri fantaśá & .554 & .461 & .123 \\
Iri preocupación empática & -1.254 & .648 & $-.239^{*}$ \\
EQ-i intrapersonal & -1.061 & .693 & -.135 \\
EQ-i interpersonal & -2.325 & .972 & $-.282^{*}$ \\
EQ-i manejo estrés & -2.374 & .953 & $-.236^{*}$ \\
EQ-i adaptabilidad & .298 & .849 & .031 \\
\hline
\end{tabular}

Nota. $\mathrm{R} 2=.415$ para paso $1 ; \Delta \mathrm{R} 2=.133$ para paso $2(\mathrm{ps}<.002)$.

${ }^{*} \mathrm{p}<.05 . \quad * * \mathrm{p}<.005$.

En suma, los resultados del análisis de regresión jerárquica ponen de manifiesto que la Inadecuación Social está predicha, de forma independiente, por cada uno de los conjuntos de variables relativas a la empatía, en concreto por las variables Toma de perspectiva $(\beta=-.231, p=$. 039) y Preocupación empática $(\beta=-.239, p=.048)$; y por la inteligencia emocional, con las variables de Factor interpersonal $(\beta=-.282, p=.020)$ y Manejo del estrés $(\beta=-.236, p=.015)$. Además, el hecho de que en el segundo paso se mantengan como variables predictivas las variables relativas a la empatía indica un posible efecto causal de la empatía sobre la inteligencia emocional.

\section{Discusión}

A la vista de los resultados, las variables que muestran una relación significativa o contribuyen de modo significativo a la explicación de la inadecuación social en una muestra de estudiantes de Educación Secundaria Obligatoria, giran en torno a los aspectos de la inteligencia emocional y la empatía, específicamente los factores de Toma de perspectiva (IRI), Preocupación empática (IRI), Factor interpersonal (EQ-i) y Manejo del estrés (EQ-i).

En base a los numerosos estudios que indican la potencial utilidad de la inteligencia emocional en las escuelas para la mejora de la competencia social y de los problemas de comportamiento (Eisenberg \& Fabes, 2006; Elias et al., 2000; Gil-Olarte et al., 2006; Lopes \& Salovey, 2004; Loukas \& Prelow, 2004), los resultados de nuestro trabajo muestran la importancia de la educación emocional y el trabajo de las habilidades sociales en la escuela (Elias et al., 2000; Gil-Olarte et al., 2006; Lopes y Salovey, 2004; Pool, 1997), ya que es un ambiente en el que aparecen numerosas demandas referidas a la competencia social (Bernart, 2006; López, 2006; Topping et al., 2000).

En la misma línea, Mestre, Guil, Lopes, Salovey y Gil-Olarte (2006) recogen tres aspectos clave sobre cómo las habilidades emocionales pueden contribuir a la adaptación social y académica del estudiante: a) el trabajo en la escuela y el desarrollo intelectual requiere la habilidad de usar y regular emociones para facilitar el pensamiento, aumentar la concentración, controlar el comportamiento impulsivo, actuar de forma efectiva bajo estrés y poseer motivación intrínseca; b) la adaptación 
social en la escuela implica establecer relaciones armoniosas con los compañeros y profesores, y las habilidades sociales juegan un papel crucial en la interacción social; c) la adaptación social en la escuela y el rendimiento académico pueden fortalecer mutuamente y además pueden aumentar la motivación de los estudiantes para implicarse en el trabajo escolar y desarrollar relaciones con los compañeros.

Nuestros resultados coinciden con varios estudios que indican una relación entre empatía y competencia social (Garmez, 1996; Howes et al., 1994; Juntilla et al., 2006; Simco y Joan, 2000), entre inteligencia emocional y empatía (Extremera y Fernández-Berrocal, 2004; Goleman, 2005; Boyatzis, 2005; Muncer y Ling, 2006; Miville et al., 2006; Petrides y Furnham, 2001) y entre inteligencia emocional y competencia social (Eisenberg y Fabes, 2006; Guil et al., 2006; Loukas y Prelow, 2004; Mestre et al., 2004; Ortega, 1997; Ortega y Lera, 2000; Petrides y Furnham, 2001).

El objetivo que se desprende de las diferentes normativas que regulan el proceso educativo en Educación Secundaria Obligatoria en España, hace referencia a un desarrollo integral de los alumnos, pero la práctica y la realidad docente parecen poner de manifiesto que en esta etapa educativa no se dedica demasiado tiempo a tratar estos aspectos. Sin embargo, la escuela es un ambiente en el que aparecen numerosas demandas referidas a la competencia social (Bernart, 2006; López, 2006; Topping, Bremner y Holmes, 2000). Se hace necesario, por tanto, revisar qué se enseña en las escuelas y proponer nuevas alternativas de actuación (Ortega y Mora-Merchan, 1996; Sastre y Moreno, 2002).

En conjunto, en nuestro trabajo se han identificado un conjunto de variables que están directamente implicadas en la Inadecuación social. La extensión de este trabajo a otros participantes y a otro contexto instruccional puede consolidar los resultados obtenidos y soslayar las posibles limitaciones del mismo.

\section{Referencias}

Bar-On, R. (1997). BarOn Emotional Quotient Inventory Technical Manual. Toronto: Multi-Health Systems.

Bar-On, R. (2000). Emotional and social intelligence: Insights from the Emotional Quotient Inventory (EQ-i). En R. Bar-On y J.D.A. Parker (Eds.), The handbook of emotional intelligence: Theory, development, assessment, and application at home, school, and in the workplace. (pp. 363-387). San Francisco, CA: Jossey-Bass Inc.

Baron-Cohen, S. y Wheelwright, S. (2004). The Empathy Quotient: An Investigation of Adults with Asperger Syndrome or High Functioning Autism, and Normal Sex Differences. Journal of Autism and Developmental Disorders, 34 (2),163-175

Bernart, M.E. (2006). It's time we teach social-emotional competence as well as we teach academic competence. Reading and Writing Quarterly Overcoming Learning Difficulties, 22(2), 103-119.

Boyatzis, R.E. (2005). Developing leadership through emotional intelligence. En C. L. Cooper y G.A. Stamatios (Eds.), Research companion to organizational health psychology. (pp. 656-669). Northampton: Edward Elgar Publishing.

Caruso, D.R. y Mayer, J.D. (1998). A Measure of Emotional Empathy for Adolescents and Adults. Unpublished Manuscipt.

Castejón, J.L., y Navas, L. (1992). Determinantes del rendimiento académico en la enseñanza secundaria. Un modelo causal. Análisis y Modificación de Conducta, 18(61), 697-730.

Cecconello, A.M. (1999). Competência social, empatia e representaçâo mental da relaçâo de apego em familias em situaçâo de risco. Tesis no publicada. 
Cecconello, A.M. y Koller, S.H. (2000). Competência social e empatia: um estudo sobre resiliência com crianças em situaçâo de pobreza. Estudos de Psicologia, 5(1), 71-93.

Coble, H. M., Gantt, D.L. y Mallinckrodt, B. (1996). Attachment, social competency, and the capacity to use social support. En G. R. Pierce, B.R. Sarason y I.G. Sarason (Orgs.), Handbook of social support and the family (pp. 141-172). Nueva York: Plenum Press.

Cohen, J., y Cohen, P. (1983). Applied multiple regression correlation analysis for the behavior sciences. Hillsdale, NJ: LEA.

Darlington, R.B. (1990). Regression and linear models. Nueva York: MCGraw-Hill.

Davis, M.H. (1983). Measuring individual differences in empathy: Evidence for a multidimensional approach. Journal of Personality and Social Psychology, 44, 113-126.

Del Prette, Z.A.P. y Del Prette, A. (1999). Psicologia das habilidades sociais: terapia e educaçâo. Rio de Janeiro: Vozes.

Eisenberg, N, Fabes, R.A. y Murphy, B.C. (1996). Parents' reactions to children's negative emotions: relations to children's social competence and comforting behaviour. Child Development, 67, 2227-2247.

Eisenberg, N. y Fabes, R.A. (2006). Emotion regulation and childrens Socioemotional Competence. En: L. Balter y C. Tamis-LeMonda (Eds.), Childs psychology: A handbook of contemporary issues (pp.357-381). Nueva York: Psychology Press.

Elias, M.J., Chan, A.Y.C. y Caputi, P. (2000). A critical evaluation of the emotional intelligence construct. Personality and Individual Differences, 28(3), 539-561.

Extremera, N. y Fernández-Berrocal, P. (2004). Inteligencia emocional, calidad de las relaciones interpersonal y empatía en estudiantes universitarios. Clínica y Salud, 15(2), 117-137.

García, M. (2003). Entrenamiento en competencias. Encuentros en Psicología Social, 1(3), 27-32.

Garmezy, N. (1996). Reflections and commentary on risk, resilience, and development. En R.J. Haggerty, L.R. Scherrod, N. Garmezy y M. Rutter (Coords.), Stres, risk, and resilience in children and adolescents: processes, mechanisms, and interventions (pp. 1-18). Cambridge: Cambridge University Press.

Gil-Olarte, P., Palomera, R. y Brackett, M.A. (2006). Relating emotional intelligence to social competence and academic achievement in high school students. Psicothema, 18(supl.), 118123.

Goleman, D. (1995). Emotional Intelligence. New York, England: Bantam Books, Inc.

Goleman, D. (1998). Working with emotional intelligence. Santa Ana: Books on Tape, Inc.

Goleman, D. (2005). Emotional Intelligence. New York: Knopf Pulishing Group.

Guil, R., Gil-Olarte, P., Mestre, J.M. y Nuñez, I. (2006). Inteligencia Emocional y adaptación socioescolar. Revista Electrónica de Motivación y Emoción, 9(22). http://reme.uji.es

Hogan, R. (1969). Development of an empathy scale. Journal of Consulting and Clinical Psychology, 33, 307-316.

Howes, C., Matheson, C.C. y Hamilton, C.E. (1994). Maternal, teacher, and child care history correlates of children's relationships with peers. Child Development, 65, 264-273.

Juntilla, N., Voeten, M., Kaukiainen, A. y Vauras, M. (2006). Multisource assessment of Children's social competence. Emotional and Psychological Measurement, 66(5), 874-895.

Kliewer, W. (1991). Coping in middle childhood: relations to competence, type A behaviour, monitoring, blunting, and locus of control. Developmental Psychology, 27, 689-697. 
Le Boterf, G. (2001). Ingeniería de las competencias. Barcelona: Gestión 2000.

Levy-Leboyer, C. (1997). Gestión de las competencias: cómo analizarlas, cómo evaluarlas, cómo desarrollarlas. Barcelona: Gestión 2000.

Lopes, P.N. y Salovey, P. (2001). Emotional intelligence and social-emotional learning: assessing emotional intelligence and developing skills and flexibility. CEIC Review, 22, 12-13.

Lopez, D. (2006). The social competence of homeschooled and conventionally schooled adolescents: A preliminary investigation. Dissertation Abstracts International: Section B: The Sciences and Engineering. Vol 66(7-B) 2006, 3974.

Loukas, A. y Prelow, H.M. (2004). Externalizing and internalizing problems in Low-Income Latino Early Adolecents: Risk, resource and Protective factors. Journal of Early Adolescence, 24(3), 250-273.

Matson, J.L., Rotatori, A.F. y Helsel, W.J. (1983). Development of a rating scale of measure social skills in children: The Matson Evaluation of Social Skills with Youngsters (Messy). Behaviour Research and Therapy, 21, 335-340.

Matthews, G., Zeidner, M., \& Roberts, R. (2003). Emotional intelligence: Science and Myth. Cambridge: MIT Press.

Mayer, J., Caruso, DR. y Salovey, P. (2000). Selecting a measure of emotional intelligence: The case of ability scales. En R. Bar-On y J. Parkes (Eds.), The handbook of emotional intelligence: Theory, development, assessment, and application at home, school, and in the workplace (pp. 320-342). San Francisco: Jossey-Bass/Pfeiffer.

Mayer, J., Caruso, DR., Salovey, P. y Sitarenios, G. (2003). Measuring emotional intelligence with the MSCEIT V2.0. Emotion, 3(1), 97-105.

McCrae, R.R. (2000). Trait psychology and the revival of personality and culture studies. American Behavioral Scientist, 44(1), 10-31.

Mehrabian, A. y Epstein, N. (1972). A measure of emotional empathy. Journal of Personality, 40, 525-543.

Méndez, F.X., Hidalgo, M.D. e Inglés, C.J. (2002). The Matson Evaluation of Social Skills for Youngsters: Psychometric properties of the Spanish translation in the adolescent population. European Journal of Psychological Assessment, 18, 30-42.

Mestre, J.M., Guil, R., Lopes, P., Salovey, P. y Gil-Olarte, P. (2006). Emotional intelligence and social y academic adaptation to school. Psicothema, 18 supl., 112-117.

Mestre, J. M., Palmero, F. y Guil, R. (2004). Inteligencia emocional: una explicación integradora desde los procesos psicológicos básicos. En J. M. Mestre y F. Palmero (Coords.), Procesos psicológicos básicos (pp. 249-280). Madrid: McGraw-Hill.

Miville, M.L., Carlozzi, A.F., Gushue, G.V., Schara, S.L. y Veda, M. (2006). Mental health counselor qualities for a diverse clientele: Linking empathy, universal- diverse orientation and emotional intelligence. Journal of Mental Health Counseling, 28(2), 151-165.

Muncer, S.J. y Ling, J. (2006). Psychometric analysis of the empathy quotient (EQ) scale. Personality and Individual Differences, 40, 1111-1119.

Ortega, R. (1997). El proyecto Sevilla anti-violencia escolar. Un modelo de intervención preventiva contra los malos tratos entre iguales. Revista de Educación, 313, 143-161.

Ortega, R. y Lera, M.J. (2000). Seville Anti-Bulling School Project. Aggressive Behavior, 26(1), 113-123.

Ortega, R. y Mora-Merchán, J. (1996). El aula como escenario de la vida afectiva y moral. Cultura y Educación, 3, 5-18. 
Pérez-Albéniz, A., De Paúl, J., Etxeberría, J., Montes, M.P. y Torres, E. (2003). Adaptación de Interpersonal Reactivity Index (IRI) al español. Psicothema, 15(2), 267-272

Petrides, K.V. y Furnham, A. (2001). Trait emotional intelligence: psychometric investigation with reference to established trait taxonomies. European Journal of Personality, 15, 425-448.

Pool, C.R. (1997). Up with emotional health. Emotional Leadership, 54(8), 12-14.

Salovey, P. y Mayer, J.D. (1990). Emotional intelligence. Imagination, Cognition, and Personality, 9, 185-211.

Sastre, G. y Moreno, M. (2002). Resolución de conflictos y aprendizaje emocional: Una perspectiva de género. Barcelona: Gedisa.

Schulze, R. y Roberts, R.D. (2005). Emotional Intelligence: An International Handbook. Cambridge: Hogrefe \& Huber Publisheres.

Simco, M. y Joan, A. (2000). Differences among peer status groups on levels of loneliness, social competence and empathy. Dissertation Abstracts International: Section B. 61(2-B).

Sternberg, R.J., Castejón, J.L., Prieto, M.D., Hautamäki, J., y Grigorenko, E. (2001). Confirmatory factor analysis of the Sternberg Triarchic Abilities Test in Three International Samples: An empirical test of the Triarchic Theory. European Journal of Psychological Assessment, 17, 1-16.

Thornton, S. y Thornton, D. (1995). Facets of empathy. Personality and Individual Differences, 19, 765-767.

Topping, K., Bremner, W. y Holmes, E.A. (2000). Social Competence. The social construction of the concept. En R. Bar-On y J. Parkes (Eds.), The handbook of emotional intelligence: Theory, development, assessment, and application at home, school, and in the workplace (pp. 28-39). San Francisco: Jossey-Bass/Pfeiffer.

Tyler, F. (1984). El comportamiento psicosocial, la competencia psicosocial individual y las redes de intercambio de recursos como ejemplos de psicología comunitaria. Revista Latino-Americana de Psicología, 16, 77-92.

Ugarriza, N. (2001). La evolución de la inteligencia emocional a través del Inventario de Bar-On (I-CE) en una muestra de Lima metropolitana. Lima (S/Ed).

Zeidner, M., Matthews, G., Roberts, R.D. (2004). Emotional intelligence in the workplace: A critical review. Applied Psychology. An International Review, 53(3), 371-399.

Zigler, E. y Trickett, P.K. (1978). IQ, social competente, and evaluation of early childhood intervention programs. American Psyhologist, 33, 789-798. 